\title{
Application and technologies for textile sensors production used in pressure distribution measurement - a critical review
}

\author{
Daniela Sofronova ${ }^{1, *}$, \\ ${ }^{1}$ Technical University of Sofia, Department of Textiles Technologies, 1000 Sofia, Bulgaria
}

\begin{abstract}
The application of smart textile sensors is continuously expanding with rising new functionalities. Their main task is to report and measure various environmental or human body parameters, such as temperature, humidity, pressure, $\mathrm{pH}$, etc., with direct contact. In this paper an in-depth research and analysis of current developments in the field of textile sensors, designed to report and measure the pressure distribution is made. Application examples in various fields of human activity are presented. Technologies and materials for their production are summarized as well as electronic components for the measurement system designs. The results of the study will be used to develop a new application for these types of sensors to help protecting human health.
\end{abstract}

\section{Introduction}

Measuring the pressure distribution of human body is an important indicator for control and prevention of various diseases. Different products with pressure measurement options are available on the market, such as mattresses, car mat, chair mat, foot mat, etc. Worldwide three commercial pressure measurement systems are available: Tekscan Body Pressure Measurement System, Vista Medical Force Sensing Array and Xsensor Full Body Sensing, but their main disadvantage is the high price.

Actually, smart textiles have the potential to measure various parameters, including pressure distribution. Nowadays textile-sensor systems could be found in our daily life for medical and safety purposes, in the entertainment and wellness [1,2]. The basic requirements for them are to provide comfort without disturbing the natural movements, sufficient measurement precision, durability and safety. According [3], smart textiles are divided on passive and active depending on that whether they are only sensing or are simultaneously sensing and acting upon detected signals.

Several technologies, summarized in $[1,3,4]$, have been developed to manufacture pressure sensors. Some authors apply a conductive material on the textile structure using different technologies as machine embroidering or sewing [5-9]. Another way is embedding the conductive material directly into the textile structure through knitting and weaving technologies [10-23]. The pros and cons of each technology are still being clarified.

\footnotetext{
* Corresponding author: dcholeva@,tu-sofia.bg0
} 
The purpose of the article is an in-depth research and analysis of current developments in the field of textile pressure sensors. The results of the study could be used to develop new kinds for these types of sensors.

\section{Technologies and application for wearable textile pressure sensors production}

The implementation of smart materials can be done at any stage of the textile production technology - spinning, weaving, knitting, printing, embroidery, chemical processing. The designs of textile pressure sensors published in scientific journals by applying various technologies are considered below, but only those applying conductive threads.

\subsection{Types of textile pressure sensors}

Three types of textile pressure sensors are known - resistive, capacitive and piezo-resistive [25]. Resistive measurement is based on material geometric strain which cause increasing of the resistivity of conductive path. In piezo-resistive sensor are used Ni or Pt alloys where two effects are added and resistivity is growing up to more than three times as large due to geometry effects alone.

Capacitance measurements are as simple as resistance measurements and also less power consuming with a higher accuracy. By capacitive sensors a deformable insulating layer is used, which separates the two conductive layers. By applying of force the distance between two conductive layers is changed, which results in a change of the electrical field between the electrodes and therefore in a change of the capacity of the sensor. The measurements of these two physical quantities require different electronic components.

\subsection{Production technologies}

\subsubsection{Machine embroidering}

Machine embroidery technology currently allows the application of two methods for textile sensor production. The first is by using a conductive thread for formation of different stitch pattern. In the second method, known as TFP method, the conductive thread is laid on the fabric and sewn with another non-conductive thread in certain places. A guide device is quite similar to those for cords guiding. The advantage of the second method is that the requirements for good sewability of the conductive thread (strength, flexible, thickness) drop out.

In [5] a capacitive pressure sensor for muscle activity recognition, consisting of 3 layers -2 electrodes and 1 dielectric, is presented. The electrodes are made by embroidering with conductive silver coated threads an array of small filled objects (1,8,16 and 30) with dimensions $2 \times 2 \mathrm{~cm}$. The achieved accuracy is $4 \%$ by range $0-10 \mathrm{~N} / \mathrm{cm}^{2}$. Figure 1 shows a sample of the embroidered electrodes. The achieved range of the sensor is the result of the correct selection of the dielectric. In addition to the desired range, the dielectric must have good elasticity, linear behaviour by loading and if possible to provide comfort. The authors use layers of a textile spacer fabric manufactured by Mueller Textil Germany, which is characterized with a thickness of $3 \mathrm{~mm}$ and a compressibility of $50 \%$ at $1.8 \mathrm{~N} / \mathrm{cm}^{2}$. The author's research shows that the hysteresis behaviour of the spacer introduce error up to $30 \%$. 


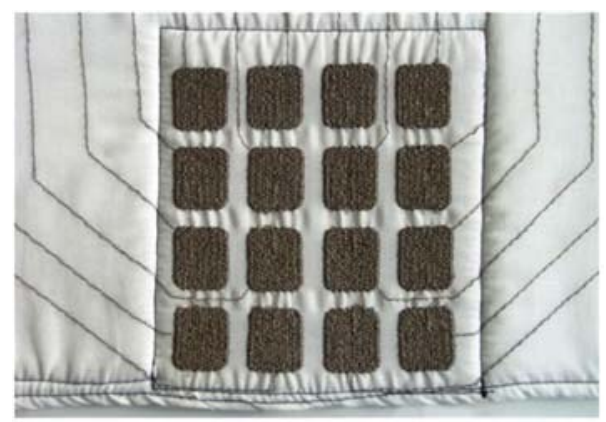

Fig. 1. Capacitive textile pressure sensor with 16 embroidered zones with silver coated threads [5].

In [6] a capacitive pressure sensor is designed for measuring pressure distribution on the human sitting position. The common electrode is a silver-coated woven textile and the second electrode is an array consisting of $2 \times 2 \mathrm{~cm}$ objects embroidered at $0,7 \mathrm{~mm}$ distance. Here as a dielectric is also used the knitted spacer fabric of Mueller Textil. The authors applied the Preisach model to quantify the sensor hysteresis and they reported a standard error below $5 \%$ for cyclic pressure change up to duration of $2 \mathrm{~h}$.

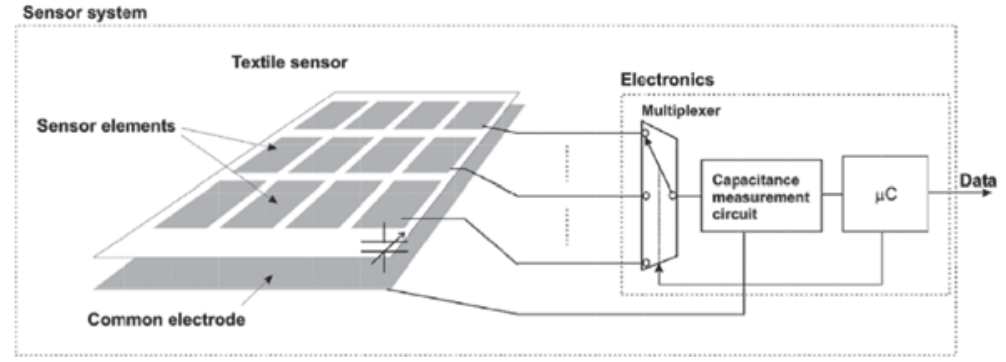

Fig. 2. Scheme of capacitive textile pressure sensor [6].

Two examples of resistive embroidered sensors are presented in [7, 8]. In [7] the authors had investigated the impact of design pattern and fabrication parameters. They reported that the textile embroidery sensors have very low activation threshold, as well as good dynamic range, signal-to-noise ratio, and part-to-part repeatability.

A multilayer textile sensor that senses a touched position is presented in [8]. It is composed of potentiometric resistive embroidery with the Ag-coated thread, an MCEY circuit pattern and a spacer fabric. The authors studied three sizes of embroidery objects $2 \times 2,3 \times 3$, and $4 \times 4 \mathrm{~mm}$ and they observed the overall contact resistance lower than 0,15 , The contact area is enough for sufficient finger touch sensing. The smaller MCEY satin stitches of the textile exhibit lower contact resistance, which is more desirable for use.

Another example is a piezo-resistive multi-touch sensing fabric that senses pressure as well as touched position [9]. Conductive-polymer-infused non-woven fabric by Eeonyx was use for piezo-resistive substrate. The resistance of the piezo-resistive fabric can be detected by sandwiching it between two conductors. To sense the position and pressure of the finger touch, the MCEY can be simply arranged in parallel on both sides of the piezo-resistive fabric at right angles to each other. A $5 \mathrm{~mm}$ spacing was chosen to estimate the finger position with sufficient resolution to capture a vibrato-like gesture. 


\subsubsection{Weaving}

In [10] a textile touch and pressure sensor prototype of woven fabric with integrated flexible thermoplastic silicones optical fibres is report. The working range is between 0 and $30 \mathrm{~N}$ with accuracy from 0,2 to $4,6 \%$. The diameter of the fibres could be in the range of 0,2 to $1 \mathrm{~mm}$ and they are characterized by good light transmission for short distances. The authors reported a lateral resolution of $10 \mathrm{~mm}$ in case of $2 \times 2$ sensor array. They also noted that the design of the sensor matrix does not allow shape recognition of an object or multitouch sensitivity, because the sensor and the signal transmission element are combined in one entity.

Implementation of conductive and electrode threads into the single layer woven structure is done in [11]. The conductive yarns are fabricated by impregnation of nonconductive wool yarns into carbon nanotubes dispersed methyl ethyl ketone. The electrode threads are made of 70 deniers of 10 galvanized nylon plated yarn twisted with a $140 \mathrm{D}$ metal plated gold yarn.

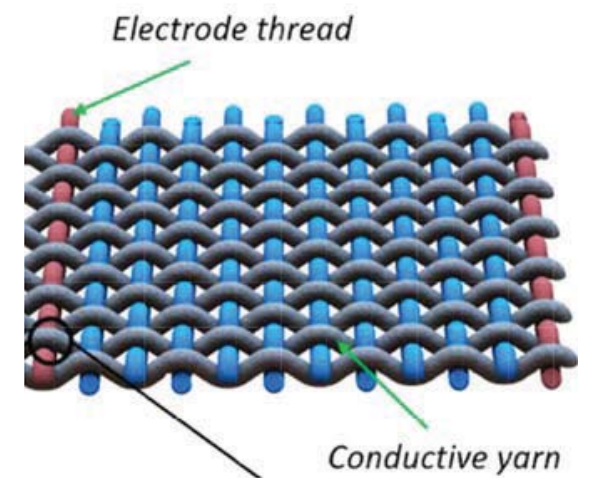

Fig. 3. Scheme of woven pressure sensor [11].

The resulting woven pressure sensors are $14 \times 14 \mathrm{~mm}$ in size and thickness $0,4 \mathrm{~mm}$. The authors report that the entire sensor area is sensitive. The studied characteristics of the sensors are: electrical resistance from $283 \Omega$ at the unload pressure to $158 \Omega$ at the load pressure; time response $50 \mathrm{~ns}$; small hysteresis $5.5 \%$; high durability under 20,000 working cycles and 50 washing cycles.

In [12] the woven sensor fabric is composed of yarns coated with organic conductive polymer with thickness of hundreds of $\mathrm{nm}$ to several $\mu \mathrm{m}$. The pressure woven is $16 \times 16 \mathrm{~cm}$ in size and has sensitivity from 0.98 to $9.8 \mathrm{~N} / \mathrm{cm}^{2}$.

Another example for woven based wearable sensor is presented in [13]. The design sensor is constructed on sandwich principle of two conductive woven fabric sheets (Shieldex NoraDell) and low density polyethylene sheet between them with a $0,1016 \mathrm{~mm}$ carbon layer and surface resistivity of 104 to $106 \Omega$. Woven fabric sheets have good conductivity and radiation efficiency characteristics, a surface resistance lower than 0,009 $\Omega / \mathrm{sq}$ and a maximum thickness of $0,13 \mathrm{~mm}$. This sandwich combination creates a piezoresistive effect that transforms the pressure into resistance variation. The sensor shows a linear behaviour of resistance of $2.2 \mathrm{k} \Omega$ to $0.41 \mathrm{k} \Omega$ to applied pressure from 1 to $70 \mathrm{kPa}$.

In [14] the sensing matrix consists also of three layers. The conductive layers are woven with conductive stripes each of them is separated with non-conductive polyethylene terephthalate. The middle layer is a pressure sensitive semi-conductive fabric. High resolution of $1 \mathrm{~cm}^{2}$, high sensitivity and wide dynamic range from $0.25 \times 10^{5}$ to $5 \times 10^{5} \mathrm{P}$ are established. 
Woven textile electrode is in the basis for development of soft tactile sensor able to detect both normal and tangential forces [15]. Capacity sensor has high sensitivity less than $10 \mathrm{mg}$ within the wide range up to $27 \mathrm{~N}$ or $400 \mathrm{kPa}$ for normal forces and for tangential forces from $0,5 \div 1,8 \mathrm{~N}$.

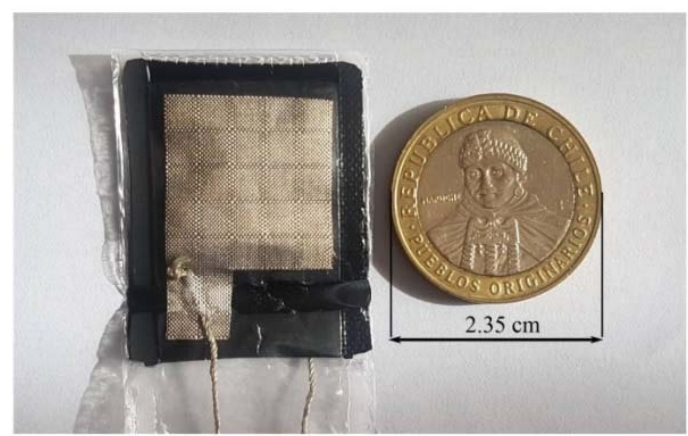

Fig. 4. Fabricated woven pressure resistive sensor [13].

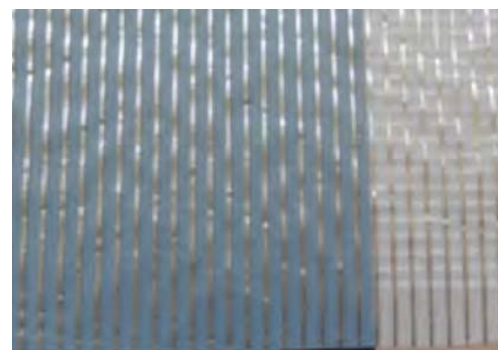

Fig. 5. Textile pressure matrix [14].

The authors in [16] design highly sensitive, self-powered and wearable electronic skin based on a pressure-sensitive nanofiber woven fabric sensor fabricated by weaving PVDF electrospun yarns of nanofibers coated with PEDOT. The sensor show high sensitivity $18,376 \mathrm{kPa}^{-1}$ at $\sim 100 \mathrm{~Pa}$, wide pressure range $0,002-10 \mathrm{kPa}$, fast response time $15 \mathrm{~ms}$ and durability 7500 cycles.

\subsubsection{Knitting}

Knitting technologies for smart textile also continues to evolve as it allows the combination of conductive and non-conductive yarns by different techniques. There are many examples for developed textile pressure sensors. In [17] are produced and tested three types of knitted pressure sensors differing in the way of arranging the stitches - Figure 6. All samples are knitted from an acrylic copper coated spun yarn and a cotton yarn plated with polyamide or elastane. The authors found that all three types of sensors show different behaviour. The first (in left) type has highest sensitivity, and the third (in right) - a uniform sensitivity. Better washability has the second type (in the middle). The last two types are suitable for dynamic loads according the authors. 
a)
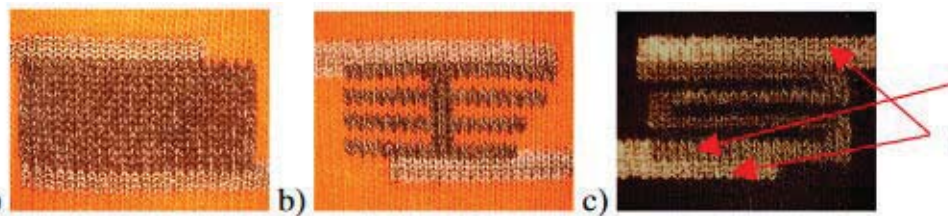

Sensor

Conductive lines

Fig. 6. Knitted textile pressure sensors [17].

Other authors studied the three types of textile sensors - resistive, piezo-resistive, and capacitive, knitted with various conductive yarn geometries [18]. They found that the resistance of knitted fabric can be program controlled. The resistive and capacitive sensors show good linear relationship between resistance and elongation.

In [19] a wearable pressure sensor knitted with conductive blended yarn in plain knit is presented. The knitted sensor demonstrates high sensitivity $0,73 \mathrm{kPa}^{-1}$ for a wide range of pressure from nearly zero to $100 \mathrm{kPa}$ and a very short response time, less than $0,4 \mathrm{~s}$. A similar example is presented in [20] for knitted sensor with a silver-plated conductive yarns. The resistive yarn path has S-shaped form as could be seen on Figure 7.

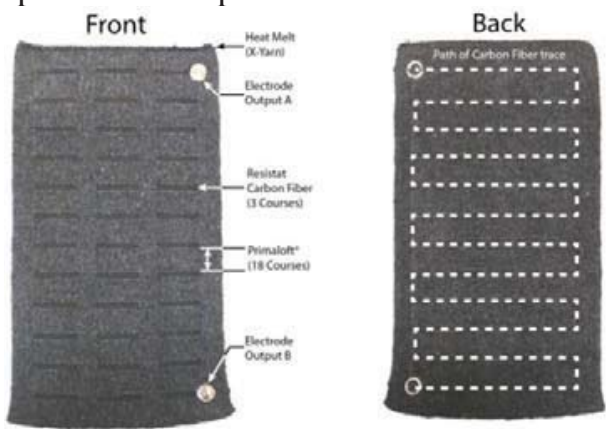

Fig. 7. Knitted sensor [19].

In paper [21] knitted fabric made from braiding silver-plated conductive yarn and nonconductive yarn with different knits are used as pneumatic artificial muscle for robotic applications. They could be working up to $300 \mathrm{kPa}$ and provide durability and high sensibility.

Two piezo-resistive knitted sensors with dimension 60x40mm together with a Velostat intermediate layer are part of developed pressure sensor described in [22]. Steel and copper conductive yarn are used, which have showed different resistance variation by loading. By steel yarn it is $3,2 \mathrm{kOhm}$ by $4583 \mathrm{~N} / \mathrm{m}^{2}$, while by the copper wire $-140 \mathrm{Ohm}$. Despite the greater resistance the steel wire has lower power consumption.

\subsection{Applications}

Wearable pressure textile based sensors for pressure detection or pressure distribution could be applied in various fields. According to the pressure range the textile pressure sensors are divided into two groups:

- Low pressure range $<10 \mathrm{kPa}$

- $\quad$ High pressure range up to $100 \mathrm{kPa}$

Sensors with low pressure range could be used as touch detectors for human-computer interaction using textile keypads [26] and the development of artificial hands for handling objects [24]. Back in 2001 an idea for replacing the traditionally packaged interfaces with textile sensors in [29]. 
Textile sensors with high pressure range for monitoring the foot pressure distribution, the hand stress during movements of heavy weights and human posture. Knowledge of pressure distribution could be applied in two ways:

- Design of new products subject to pressure - footwear, medical stockings, matrasses, mats, chairs, etc.

- Prevention by online continuous measurement and control of pressure.

Human posture could be measure by gaming; car driving; daily activities; sport activates like cycling; sitting, laying and standing poses wheelchairs and hospital beds for prevention of pressure ulcers, rehabilitation after a stroke, requires the controlled motion of limbs [27]. Concept of a "Sensitive Skin" based on a large-area, flexible arrays, also consists of pressure sensors and is presented in [28]. Textile gloves monitoring the hand stress during manual activity are described in [30].

\section{Conclusion}

The wearable pressure sensors should fulfilled requirements as good ergonomics, allowing natural movements and a sensor response in a broad range of pressure to allow a large variety of possible activities to be monitored. Therefore many solutions exist. Some of them are expensive high-tech wearable devices and the other part are demonstration projects with undefined reliability. The implementation of smart materials can be done at any stage of the textile production technology, but most used are weaving, knitting and embroidering. Embroidery techniques using conductive threads have become a very attractive approach for smart wearables because of their design freedom and ease of manufacture compared to other processes such as weaving, knitting, and printing.

The sensing of textile pressure sensors are based on resistive, capacitive and piezoresistive effects. In modern sensors more perspective are capacitive and piezo-resistive technology. Suitable sensor system designs are sought depending on the patterns, sensitivity range, conductive thread consumption, component provision and reliability in wearing and washing.

This paper is funded by SRC at TU-Sofia in the framework of a project No. 201PR0005-02 "Establishment of e-textiles for pressure control on the body in bedridden patients".

\section{References}

1. V. Custodio, Herrera F.J., López G., Moreno J.I., Sensors, 12 (2012)

2. L. Possanzini, M. Tessarolo, L. Mazzocchetti, E. G Campari, B. Fraboni, Impact of Fabric Properties on Textile Pressure Sensors Performance, Sensors, 19 (2019) doi:10.3390/s19214686

3. M. Stoppa, A. Chiolerio, Sensors, 14, 7 (2014)

4. C. M. A. Ashruf, Sensors Review, 22, 4 (2002)

5. J. Meyer, P. Lukowicz, G. Tröster, P. 10th IEEE Int. Symp. Wear. Comp. - Montreux, Switzerland (2006)

6. J. Meyer, B. Arnrich, J. Schumm, G. Troster, IEEE Sensors J. (2010)

7. M. Sergio, N. Manaresi, M. Tartagni, R. Guerrieri, R. Canegallo, IEEE Sensors J. (2002)

8. R. Aigner, A. Pointner, T. Preindl, P. Parzer, M. Haller, CHI '20, (2020) 
9. Jung-Sim Roh, Chapter in Book Wearab. Tech., http://dx.doi.org/10.5772/intechopen.76627

10. M. Rothmaier, M. P. Luong, F. Clemens, Sensors, 8 (2008)

11. G. Kim, C. C. Vu, J. Kim, Appl. Sci., 10 (2020)

12. S. Takamatsua, T. Kobayashi, N. Shibayama, K. Miyake, T. Itoh, Sens. Actuators A Phys., 184 (2012)

13. F. Pizarro, P. Villavicencio, D. Yunge, M. Rodríguez, G. Hermosilla, A. Leiva, Sensors, 18, 4 (2018)

14. J. Cheng, M. Sundholm, B. Zhou, M. Hirsch, P. Lukowicz, Pervasive Mob. Comput., $30(2016)$

15. L. Viry, A. Levi, M. Totaro, A. Mondini, V. Mattoli, B. Mazzolai, L. Beccai, Adv. Mater., 26, 17 (2014)

16. Y. Zhou, J. He, H. Wang, K. Qi, N. Nan, X. You, W. Shao, L. Wang, B. Ding, S. Cui, Highly sensitive, self-powered and wearable electronic skin based on pressuresensitive nanofiber woven fabric sensor, P. IEEE, 7, 1 (2017)

17. N. Baribina, A. Oks, I. Baltina, P. Eizentals, Comparative analysis of knitted pressure sensors, Eng. Rural Develop. (2018)

18. J. Ou, D. Oran, D. D. Haddad, J. Paradiso, H. Ishii, 3D Print. Addit. Manuf., 6, 1 (2019)

19. X. Juan, J. Yongtang, M. Menghe, Smart Mater. Struct., 28, 3 (2019)

20. Y. Li, X. Miao, L. Niu, G. Jiang, P. Ma, Sensors, 20, 1 (2019)

21. B. Jamil, S. Lee, Y. Choi, Fabrication, P. IEEE, 7 (2019)

22. L. Capineri, Procedia Eng., 87 (2014)

23. W. Fan, Q. He, K. Meng, X. Tan, Z. Zhou, G. Zhang, J. Yang, Z. L. Wang, Appl. Sci. Eng., 6, 11 (2020)

24. L. Possanzini, M. Tessarolo, L. Mazzoccheti, E. G. Campari, B. Fraboni, Sensors, 19 (2019)

25. S. Kärki, J. Lekkala, Pressure mapping system for physiological measurements, $P$. XVIII IMEKO Brazil (2006)

26. Eleksen Ltd (accessed on 29 June 2020), available online: http://www.eleksen.com

27. P. Lukowicz, F. Hanser, C. Szubski, and W. Schobersberger, P. 4th IEEE Pervas. Comput., Ireland (2006)

28. V. J. Lumelsky, M. S. Shur, S. Wagner, IEEE Sensors J., 1 (2001)

29. S. S. Swallow, A. P. Thompson, Sensory Fabric for Ubiquitous Interfaces, Int. J. HumComput. Interact., 13, 3 (2001)

30. M. Tessarolo, L. Possanzini, E. G. Campari, R. Bonfiglioli, F. S. Violante, A. Bonfiglio, B. Fraboni, Flex. Print. Electron., 3, 3 (2018) 